(2) Open Access Full Text Article

\title{
Hydroxyapatite Nanoparticles Facilitate Osteoblast Differentiation and Bone Formation Within Sagittal Suture During Expansion in Rats
}

This article was published in the following Dove Press journal:

Drug Design, Development and Therapy

\section{Wei Liang \\ Pengbing Ding \\ Guan Li (D) \\ Enhang Lu \\ Zhenmin Zhao}

Department of Plastic Surgery, Peking University Third Hospital, Beijing, I00I9I, People's Republic of China
Correspondence: Zhenmin Zhao Department of Plastic Surgery, Peking University Third Hospital, Beijing, I00191, People's Republic of China

Email zzmbysy@sina.com
Background: The potential of relapse of craniofacial disharmony after trans-sutural distraction osteogenesis is high due to the failure to produce a stable bone bridge in the suture gap. The aim of this study is to evaluate whether hydroxyapatite nanoparticles (nHAP) have the effect of promoting osteoblast differentiation of suture-derived stem cells (SuSCs) and bone formation in sagittal suture during expansion.

Methods: SuSCs were isolated from sagittal sutures and exposed to various concentrations of nHAP $\left(0,25,50\right.$, and $\left.100 \mu \mathrm{g} \mathrm{mL}^{-1}\right)$ to determine the optimal concentration of nHAP in osteoblast differentiation via performing Western Blotting and RT-qPCR. Twenty 4-week-old male Sprague-Dawley rats were randomly assigned into 4 groups: SHAM (sham-surgery), distraction, ACS (absorbable collagen sponge) and ACS+nHAP groups. In the ACS and ACS + nHAP groups, saline solution and nHAP suspended in a saline solution were delivered by ACS placed across the sagittal suture, respectively. In the latter three groups, the suture was expanded for 14 days by $50 \mathrm{~g}$ of constant force via a W shape expansion device. Suture gap area, bone volume fraction (BV/TV) and bone mineral density (BMD) of sagittal sutures were assessed via micro-CT, while the mechanical properties of sagittal sutures were evaluated via nanoindentation test. The efficacy of nHAP on bone formation in sagittal suture was also evaluated via BMP-2 immunohistochemistry staining.

Results: The expression of osteoblast related genes and proteins induced by $25 \mu \mathrm{g} \mathrm{mL}$ nHAP were significantly higher than the other groups in vitro $(\mathrm{p}<0.05)$. Furthermore, treating with $25 \mu \mathrm{g} \mathrm{mL}^{-1} \mathrm{nHAP}$ in vivo, the suture gap area was significantly reduced when compared with the distraction group. Correspondingly, the BV/TV, BMD, hardness and modulus of sagittal sutures were significantly increased in the ACS + nHAP group $(p<0.05)$.

Conclusion: The $25 \mu \mathrm{g} \mathrm{mL}^{-1}$ dose of nHAP delivered by ACS can facilitate bone formation into the sagittal suture during expansion via inducing osteoblast differentiation of SuSCs.

Keywords: hydroxyapatite nanoparticles, suture-derived stem cells, osteoblast differentiation, sagittal suture, bone formation

\section{Introduction}

Trans-sutural distraction osteogenesis (TSDO), characterized by conducting continuous mechanical force to the immature suture, is a powerful strategy for craniofacial disharmony patients. ${ }^{1}$ After the optimal expansion, the suture undergoes remodeling with bone fiber rearrangement, cartilage formation, resorption and ossification. This process continues until the architectural environment achieves stabilization. $^{2-6}$ Nevertheless, it is well documented that, even after a relatively long period, the distended connective tissue of the suture has a strong tendency to 
rebound to its previous form, which results in relapse. ${ }^{7}$ The reason for this is that the remodeling process of suture mainly involves the ossification at lateral bony edges, and the slow rate of bone formation in the suture has been considered an obstacle to be conquered for the reduction of the relapse rate. ${ }^{8}$ It would be potentially beneficial to manipulate the key biological process through promoting bone formation and remodeling during expansion. ${ }^{9}$ To date, various methods have been developed to maintain the suture expansion and facilitate the bone regeneration, such as recombinant human bone morphogenetic protein-2 (rhBMP-2) ${ }^{10}$ vitamin $\mathrm{C},{ }^{11}$ isotretinoin, ${ }^{12}$ parathyroid hormone ${ }^{13}$ and bone marrow mesenchymal stem cells (MSCs). ${ }^{14}$

Hydroxyapatite (HAP) is a critical composition to skeleton health and is the storage material of calcium and phosphate for bone remodeling. ${ }^{15}$ Biosynthesis of hydroxyapatite nanoparticles (nHAP) during bone matrix mineralization is a controlled biological process in which differentiating osteoblasts concentrate environmental calcium and phosphate to form hydroxyapatite and then exocytose onto the type I collagen fibers interface via matrix vesicles and act as an initiator of bone formation in the bony skeleton. ${ }^{16,17}$ As such, HAP is currently being investigated as a therapeutic biomaterial for orthopedic and dental purposes. ${ }^{18}$ An nHAP is an artificially synthesized nanostructure that has excellent biocompatibility and bioactivity owing to its high chemical structural similarity to natural bone. Several studies have suggested that giving extracellular nHAP can influence osteoblast lineage commitment and cell function through releasing $\mathrm{Ca}^{2+}$ continuously. ${ }^{19}$ Indeed, recent evidence demonstrated that the hydroxyapatite nanoparticle is a remarkable osteoinductive agent on human mesenchymal stem cells due to the up-regulation of osteoblastogenic genes (alp, coll, runx2) in human mesenchymal stem cells (MSCs) grown on hydroxyapatite biomaterial. ${ }^{20}$ Furthermore, previous studies have found that the effects of nHAP on cells varied considerably depending on its concentration, size, shape and surface charge, and spherical nHAP under $80 \mathrm{~nm}$ in diameter showed more favorable features for osteoblast differentiation, which might be the well-organized surface and size beneficial for filopodia protrusion of osteoblasts cells. $^{21-23}$

It has long been well known that the suture-derived mesenchyme stem cells, termed $\mathrm{SuSCs},{ }^{24}$ in the niche have the potential to contribute to calvarial morphogenesis, bone regeneration and injury repair. ${ }^{13,24,25}$ Thus, current strategies use pharmaceuticals, exosomes or cytokines to induce SuSCs to osteoblast differentiation and enhance bone regeneration during the expansion process. $^{26-29}$ However, the success of these reconstructions remains highly challenging owing to a number of limitations. ${ }^{7}$ In the present study, we hypothesized that applying nHAP could prevent the expanded suture occlusion via inducing SuSCs to osteoblast differentiation and enhance local mechanical strength in certain sutures. We synthesized a spherical nHAP approximately $55 \mathrm{~nm}$ in diameter in advance and examined its anabolic effect on SuSCs. The optimal concentration of nHAP for promoting osteoblast differentiation was well determined. Next, we identified the effects of nHAP facilitating the ossification in the expanding suture on the rat model via multiple methods, including micro-CT analysis, immunohistochemistry staining and the nanoindentation test in vivo. To eliminate the effects of occlusal forces and mastication, in vivo experiments was conducted on the rats' sagittal sutures.

\section{Materials and Methods Preparation of nHAP Particles}

All chemical reagents were analytical grade and were used without any additional purification. The size-controlled nHAP were prepared according to the typical method, as described previously. ${ }^{30}$ Briefly, based on the sol-gel and hydrothermal reaction system, Hexadecyl (cetyl) trimethyl ammonium bromide (CTAB) was used to control the size of the nHAP particles. $\mathrm{CaCl}_{2}(10 \mathrm{mM})$ solution $(30 \mathrm{~mL})$ was dropped into $200 \mathrm{~mL} \mathrm{Na} \mathrm{HPO}_{4}(25 \mathrm{mM})$ in the presence of CTAB $\left(9.0 \times 10^{4} \mathrm{~mol} / \mathrm{L}\right.$ to $\left.12 \times 10^{4} \mathrm{~mol} / \mathrm{L}\right)$ in a magnetically stirred vessel at $30^{\circ} \mathrm{C}$. The $\mathrm{pH}$ value of the reaction system was maintained at $8.0 \pm 0.5$ by using a dilute ammonia solution $(0.1 \mathrm{M})$. The precipitate was filtered by a $0.22 \mu \mathrm{m}$ membrane, and was then washed completely with distilled water and ethanol to remove the residual of CTAB.

nHAP particles were characterized by dynamic light scattering (DLS, Brookhaven Instrument-Omni, USA), X-ray diffraction (XRD, Bruker D8 Advance instrument) and scanning electron microscope (S-4800, Hitachi). Prior to the cell and animal experiments, nHAP were subjected to filtration through a $0.22 \mu \mathrm{m}$ filter membrane to preclude bacteria. The crystallinity degree was calculated as the ratio between the area corresponding to the crystalline 
phase and the total area under the XRD curve using the formula: $:^{31}$

$$
C I=\frac{S_{\text {cr.phase }}}{S_{\text {total }}} \times 100 \%
$$

where $C I$ is the crystallinity degree; $S_{\text {crphase }}$ is the area corresponding to the crystalline phase; and $\mathrm{S}_{\text {total }}$ is the total area under the XRD pattern.

\section{SuSCs Cell Isolate and Culture}

Healthy Sprague-Dawley (SD) male rats (Vital Rive, Beijing, China) aged 4 weeks were used in this study. Ethical clearance was obtained from the Peking University Biomedical Ethics Committee (No. LA2019177). All the in vivo and in vitro cellular experiments were performed according to the National Institutes of Health regulations for the care and use of animals. The term suture-derived stem cells (SuSCs) was used following named guidelines for animal welfare.

The SuSCs were harvested from sagittal sutures of 4-week-old healthy Sprague-Dawley (SD) male rats by explant culture following the method described earlier. ${ }^{32}$ Briefly, the sagittal sutures with $3 \mathrm{~mm}$ bony margins were dissected with great care and minced into $1 \mathrm{~mm}^{3}$ pieces. Then, the suture explants were incubated in a standard growth medium containing Dulbecco's Modified Eagle Medium (DMEF-12; Hyclone Laboratories, Logan, UT, USA), $10 \%$ fetal bovine serum, and $100 \mathrm{IU} / \mathrm{mL}$ of penicillin/streptomycin. Suture explant samples were placed with the endocranial surface face down in the culture dish, allowing for cell migration. Explants were maintained at $37^{\circ} \mathrm{C}$ in an atmosphere of $5 \%$ carbon dioxide; the medium was changed every other day. After two weeks, in vitro cultured cells were passaged by trypsinization. SuSCs of the third time passage were used for the following experiments.

\section{Induction Adipo-Osteogenic Differentiation of SuSCs}

For osteogenic differentiation, the third time passage cells were seeded onto 48 -well plates at a density of $1 \times 10^{4}$ cells per well in accordance with the previous study. ${ }^{33}$ When cell confluence reached $80 \%$, SuSCs were treated with an osteogenic medium (DMEM containing $100 \mathrm{nM}$ dexamethasone, $50 \mathrm{mg} / \mathrm{mL}$ ascorbic acid, and $5 \mathrm{mM} \beta$ glycerophosphate (Sigma, MO, USA)) and cultured for an additional 14 days. For adipogenic differentiation, SuSCs were cultured in an adipogenesis induction medium
(DMEM containing $1 \mu \mathrm{M}$ dexamethasone, $10 \mu \mathrm{M}$ insulin, $100 \mu \mathrm{M}$ indomethacin, and $500 \mu \mathrm{M}$ isobutylmethylxanthine) for 21 days. The medium was replenished after every 3 days of differentiation. Two percent Alizarin Red ( $\mathrm{pH} 4.2$, Sigma) and oil red O solution were used to detected calcium nodule deposits and lipid droplets resulting from differentiation.

\section{Flow Cytometry}

Cell surface antigens of SuSCs were detected by flow cytometry. Isolated cells were examined for the presence of the mesenchymal stem cell markers (CD90, CD29, $\mathrm{CD} 11 \mathrm{~b} / \mathrm{c}$ ) and the absence of markers (CD73, CD34, and CD44, CD45,) as described previously, according to the criteria for defining multipotent mesenchymal stromal cells. $^{34}$ Briefly, cells were trypsinized and fixed in $4 \%$ formaldehyde solution. Primary antibodies for above cell surface markers diluted (1:100) with $1 \%$ bovine serum albumin were then applied. Second, cells were incubated with corresponding PE-conjugated secondary antibodies at $4{ }^{\circ} \mathrm{C}$ for $1 \mathrm{~h}$. Stained cells (approximately $5 \times 10^{5}$ cells per group) were analyzed by flow cytometry using a fluorescent-activated cell sorting (FACS) Calibur.

\section{Biological Evaluation of nHAP on Cell SuSCs Viability Assay after Exposure to nHAP}

The third passage cells were seeded into three 96-well plates $\left(5 \times 10^{3}\right.$ cells per well $)$ in triplicate and incubated for $24 \mathrm{~h}$. The medium was replaced by a fresh medium containing different concentrations of nHAP $(0,25,50$ and $100 \mu \mathrm{g} / \mathrm{mL}$ ), followed by incubation for another $24 \mathrm{~h}$ and $48 \mathrm{~h}$. Subsequently, the medium was removed and each cell was washed twice with PBS. Then, a fresh medium containing 1/10 (v/v) CCK-8 solution was added to each well and incubated at $37{ }^{\circ} \mathrm{C}$ for $1 \mathrm{~h}$. Finally, the absorbance was measured at $450 \mathrm{~nm}$ using a 96-well plate reader.

\section{Calcein AM/PI Test}

SuSCs were seeded into a confocal laser scanning microscope (CLSM) specific dish with a density of $70 \%$ for $24 \mathrm{~h}$. After further treating with different concentrations of nHAP $(0,25,50$ and $100 \mu \mathrm{g} / \mathrm{mL})$ for $24 \mathrm{~h}$, the cells were washed three times with PBS. Then, the cells were co-stained with Calcein-AM and propidium iodide (PI) for 5 min and washed three times with PBS. The CLSM apparatus was used to obtain the fluorescence living cell and dead cell images of the four samples. 
Effect of Nano-HAP on Osteoblast Differentiation Under Mechanical Stimuli

Recent reports have demonstrated that nHAP modulates osteoblast differentiation by regulating gene expression, including alkaline phosphatase (alp), type I collagen (coll), and RUNX family transcription factor 2 (runx2) genes. ${ }^{16,20}$ In our experiment, the expression levels of the above genes were used to estimate the osteoinductivity efficacy of nHAP on SuSCs cells under mechanical stimuli. According to previous studies, cells were co-cultured with an osteogenic medium containing different concentrations $(0$, 25,50 and $100 \mu \mathrm{g} / \mathrm{mL}$ ) of nHAP in the presence of mechanical stretch in vitro produced by the Flexcell $4000 \mathrm{~T}$ tension system $(10 \%$ at $0.5 \mathrm{~Hz}) .{ }^{35}$ The 14 days of cell culture were selected for total RNA isolation and quantification. The $2-\Delta \Delta \mathrm{Ct}$ method was used to calculate the expression values. Alizarin Red S was applied at 14 days and the mineralization rate were assessed under $562 \mathrm{~nm}$ using a 96-well plate reader. The primers used in this experiment were as follows: $A L P$, forward 5'- ATGGTAACGGGCCTGGCTACA -3'and reverse 5'- AGTTCTGCTCATGGACGCCGT -3'; COL1, forward 5'- CCAGCTGACCTTCCTGCGCC $-3^{\prime}$ and reverse 5'- CGGTGTGACTCGTGCAGCCA -3'; Runx2, forward 5'- AGCGGACGAGGCAAGAGTTT -3'and reverse 5'AGGCGGGACACCTACTCTCATA-3'; GAPDH, forward 5'- ACCACAGTCCATGCCATCAC -3'and reverse 5'TCCACCCTGTTGCTGTA-3' ${ }^{16,36}$

\section{Western Blotting}

SuSCs were seeded in 6-well plates for $24 \mathrm{~h}$ before treatment with various concentrations of $\operatorname{nHAP}(0,25,50$, and $100 \mu \mathrm{g} \mathrm{mL}^{-1}$ ) for 14 days under stretch. Cells were lysed with a RIPA lysis buffer complemented by a protease inhibitor cocktail (P8340, Sigma, USA). After suspending with an SDS loading buffer and heating at $95^{\circ} \mathrm{C}$ for $10 \mathrm{~min}, 20$ $\mu \mathrm{L}$ samples were loaded onto SDS-PAGE gel and electrotransferred to a polyvinylidene fluoride (PVDF) membrane. The PVDF membranes were blocked with TBST supplemented with 5\% nonfat dry milk at room temperature for 1 $\mathrm{h}$, and then incubated with primary antibodies at indicated concentrations at $4{ }^{\circ} \mathrm{C}$ overnight. The primary antibodies used were runx2 (\#12556S, CST), 1:1000; osteopontin (\#ab8848, abcam), 1:1000; coll (\#84336S, CST), 1:1000; and gapdh (\#2118S, CST), 1:1000. After incubation with an HRP-linked mouse anti-rabbit (\#ab99697, abcam) secondary antibody for $1 \mathrm{~h}$ at room temperature, the PVDF membranes were visualized with an ECL chemoluminescence kit (\#32,106, Thermo Fisher) and imaged using a TANON 5200 Multi (TANON, Beijing, China).

\section{Animal Model}

Suture expansion surgery was performed on 20 male rats aged 4 weeks under $2 \%$ pentobarbital sodium solution anaesthesia $(600 \mu \mathrm{g} / \mathrm{kg}$; i.p.) and the operation of sagittal suture expansion on rats adapted from a previously study. ${ }^{10}$ Briefly, after anesthetizing the rats, a dental drill was applied to make a hole (diameter $=1 \mathrm{~mm}$ ) on both sides of the sagittal suture at a distance of $12 \mathrm{~mm}$ to place the W shape expansion device made by a 0.024 -inch in diameter nickel titanium alloy wire. The initial expansion force was approximately $50 \mathrm{~g}$ (Figure 1A). Five control rats were sham performed with broken springs without force (SHAM group) and the other animals were randomly distributed into three groups: (1) springs-only group (distraction group); (2) absorbable collagen sponge (ACS, $6 \times 8 \times 2 \mathrm{~mm}$ ) was soaked with $0 \mu \mathrm{g} / \mathrm{mL}$ nHAP (ACS group); (3) absorbable collagen sponge (ACS, $6 \times 8 \times 2 \mathrm{~mm}$ ) was soaked with $100 \mu \mathrm{g} / \mathrm{mL}$ nHAP (ACS + nHAP group). At the end of the experiment (14 days), all animals were suffocated by overdose of $\mathrm{CO}_{2}$.

\section{Microcomputed Tomography (Micro-CT) Analysis}

Animal skulls were harvested and fixed in 4\% paraformaldehyde solution and scanned coronally using highresolution micro-CT (Quantum GX, PerkinElmer). Raw image data comprised three-dimensional reconstructions and two-dimensional coronal sections of the intact midsagittal suture created by software (Analyze12.0, PerkinElmer) and measured the distance between the two holes on the right and left parietal bones. The Image $\mathrm{J}$ software was applied to analyze the gap area of the central 3-mm-long portion of the sagittal suture. ${ }^{2}$ Meanwhile, the volume of newly formed bones in the expanded suture were estimated by calculating the bone mineral density (BMD) and bone volume fraction (BV/ TV) of the intact sagittal suture.

\section{Histology and Histochemistry}

After micro-CT scanning, the samples were demineralized in $15 \%(\mathrm{w} / \mathrm{v})$ ethylene diaminetetra acetic acid (EDTA) for a month, and then the decalcifying sagittal sutures were cut into $10 \mu \mathrm{m}$ slices for hematoxylin and eosin (HE) staining. To investigate the osteoinduction efficiency of nHAP, serial sections from all groups were BMP-2 
A

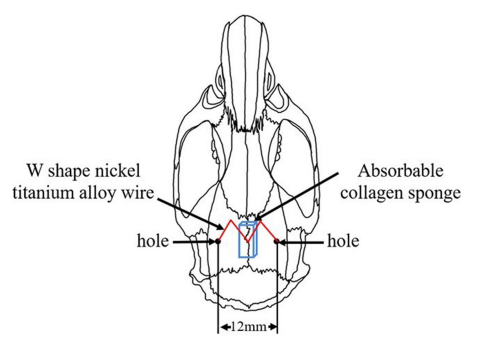

B

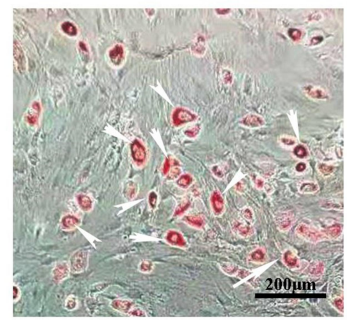

C

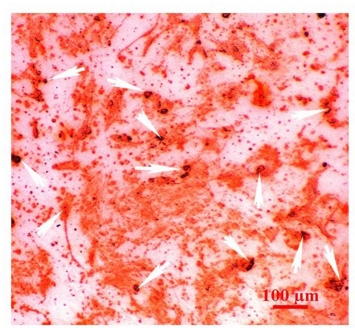

D

\section{Positive}
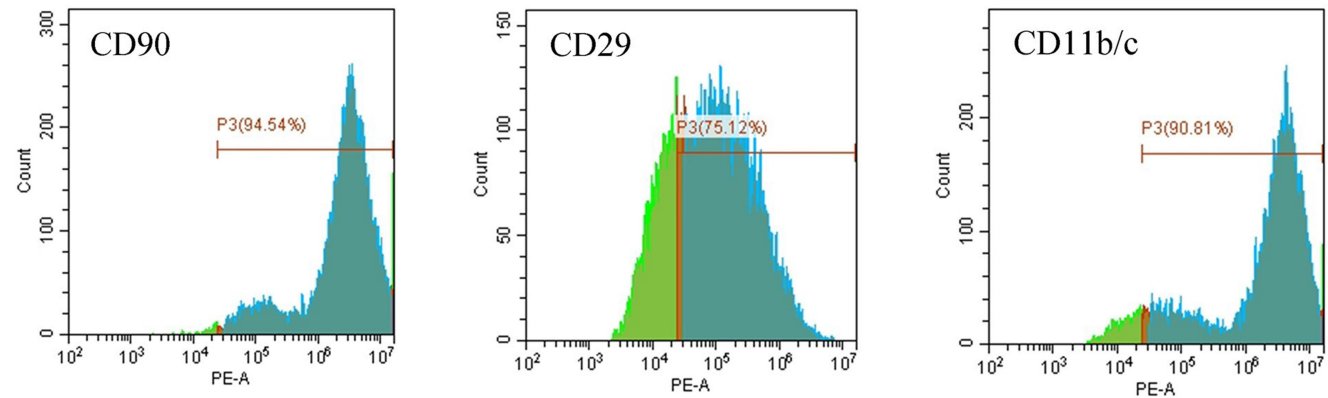

E

Negative
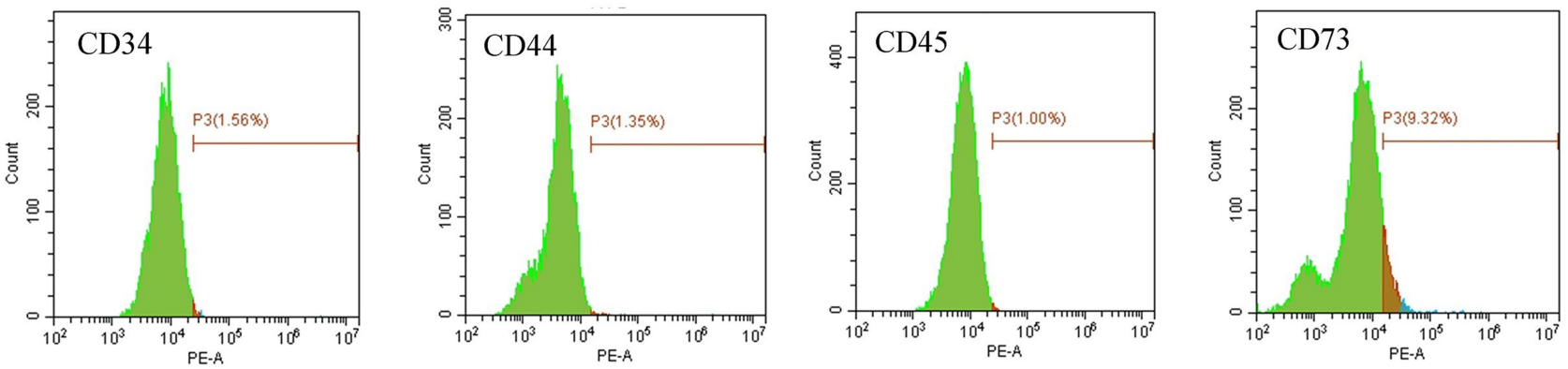

Figure I Characterization and differentiation of suture-derived stem cells (SuSCs). (A) Schematic illustration of holes made on the parietal bone to place the expansion appliance and absorbable collagen sponge. (B) Oil red staining indicated lipid restoration in SuSCs after cultured in lipid-forming medium for 21 days; white arrows indicate oil drop scale bar, $200 \mu \mathrm{m}$. (C) Alizarin red S staining of SuSCs cultured in osteogenic medium at day 14; white arrows indicate calcium nodules scale bar, $100 \mu$ m. Flow cytometry analysis shows that SuSCs are expressing markers (D) CDI lb/c, CD29 and CD90, but (E) not expressing markers CD34, CD44, CD45 and CD73.

immunohistochemistry stained according to the manufacturer's protocol.

\section{Nanoindentation Test}

Sagittal suture samples for nanoindentation testing were successively dehydrated by sequential soaking in ethanol solutions with increasing concentrations (50-70-90$100 \%$ ) and defatted in acetone for $2 \mathrm{~h}$. Then, samples were kept with their longitudinal axes positioned vertically to the ground and their lower parts embedded in epoxy resin. Samples were stored at room temperature overnight for air drying and hardening and were then cut with a low- speed diamond saw to create smooth surfaces $2 \mathrm{~mm}$ above the growth plates, perpendicular to the long axes of the sutures. The microarchitecture bony tissue in the sutures was first polished by a rotational polishing machine (Mecatech 234, Presi) with silicon carbide paper of grit sizes 320, 500, 1200, 2000 and 4000. Further fine polishing was done by micro cloths with alumina powder of grit diamonds $3 \mu \mathrm{m}$. Nanoindentation tests were conducted by Agilent G200, with a 3-sided diamond pyramid tip. The modulus $(\mathrm{E})$ and the hardness value $(\mathrm{H})$ were calculated from the slope of the unloading curve and from the indentation area, respectively. Standardized 
values for bone and indentor Poisson ratios (0.3) were used through the application of the Oliver-Pharr method. ${ }^{37,38}$ Eight indents were produced on each sample, where neighboring indents were spaced more than $10 \mu \mathrm{m}$ apart. This peak load produced a maximum indentation depth of approximately 0.8 to $1 \mu \mathrm{m}$, ensured an accurate modulus value, and reduced the effect of residual surface roughness.

\section{Statistical Analysis}

Statistical analysis was performed using SPSS ver. 20.0 software (IBM Corp, Armonk, NY, USA). Data are expressed as mean $\pm \mathrm{SD}$. The statistical significance of animal experiment data was analyzed by permutation tests. The statistical significance of other data was assessed by one-way analysis of variance (ANOVA) and $p<0.05$ was considered a statistically significant difference.

\section{Results}

\section{Characteristics of Synthesized nHAP}

The particle size distribution depicted by dynamic light scattering (DLS) was relatively narrow and the average diameters of nHAP were $54.98 \pm 4.55 \mathrm{~nm}$, as shown in Figure 2A. From the size analysis results based on FESEM images, as shown in Figure 2B, it was observed that the spherical particles of nHAP size range from $30-90 \mathrm{~nm}$, confirming that nHAP is a nanoparticle. In detail, the characteristic peaks at $2 \theta$ regions of $22.46,26.11^{\circ}$, $33.28^{\circ}$ and $39.87^{\circ}, 44.06^{\circ}, 49.23^{\circ}$, which matches the characteristic XRD spectrum of hydroxyapatite (JCPDS09-0432, Hydroxyapatite). Moreover, based on the XRD pattern, we identified that the crystallinity degree of nHAP was $76 \pm 3.6 \%$. All results indicate that the synthesized nHAP was composed of hydroxyapatite and it has a relatively higher degree of crystallinity. ${ }^{39}$

\section{Characterization of Isolated SuSCs}

SuSCs identification was done by analyzing stem cellspecific markers. Flow cytometric characterization analysis showed that the SuSCs were homogenously positive (Figure 1D) for CD90 (94.54\%), CD29 (75.12\%) and $\mathrm{CD} 11 \mathrm{~b} / \mathrm{c}(90.81 \%)$ and negative (Figure 1E) for CD34 (1.56\%), CD44 (1.35\%), CD45 (1.00\%) and CD73 (9.32\%). Lipid forming medium treatment for 21 days in vitro resulted in lipid restoration in SuSCs indicated by Oil red staining (white arrows) (Figure 1B). Consistently, OS-cultured (osteogenic medium) SuSCs indicated osteoblast characterization as evidenced by intense Alizarin red staining (white arrows, Figure 1C). These results demonstrated that SuSCs had stem-cell properties in vitro.

\section{Cell Viability Assay Live/Dead Staining}

SuSCs were treated with different concentrations $(0,25$, 50 and $100 \mu \mathrm{g} / \mathrm{mL}$ ) of nHAP for $24 \mathrm{~h}$ and $48 \mathrm{~h}$. The cytotoxicity was then also evaluated in a visualized manner by live/dead staining, where live and dead cells were differentiated by co-staining with PI (red fluorescence; dead cells) and calcein AM (green fluorescence; living cells) after laser exposure. As shown in Figure 3A, the vast majority of the SuSCs exhibited green fluorescence and only a few cells were killed and stained in red fluorescence in all nHAP containing groups, which were similar to the control group, suggesting a negligible damaging effect from nHAP. Correspondingly, the viability of SuSCs was assessed using the cell-counting kit-8 (CCK8) assay. nHAP were found to have no effect on cell viability compared to the control $(0 \mu \mathrm{g} / \mathrm{mL})$ even up to the maximum treated concentration of $100 \mu \mathrm{g} / \mathrm{mL}$ (Figures $3 \mathrm{~B}$ and $\mathrm{C}$ ). Taken together, these results
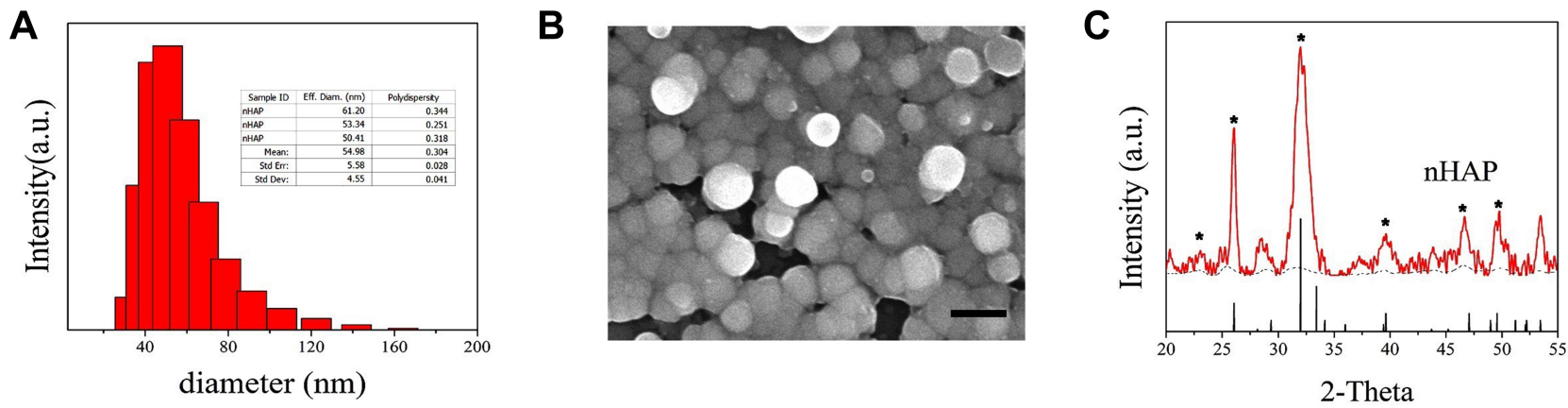

Figure 2 Nano-hydroxyapatite (nHAP) characterization. (A) DLS particle size distribution and median size of nHAP. (B) SEM image, scale bar, I00nm. (C) XRD spectrum. The characteristic peaks of hydroxyapatite are indicated by *. 

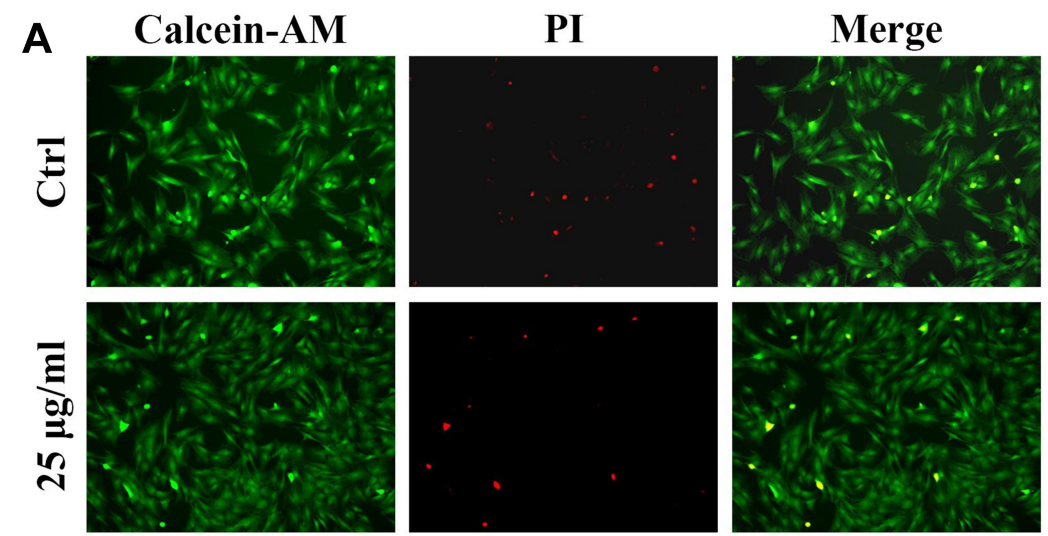

B
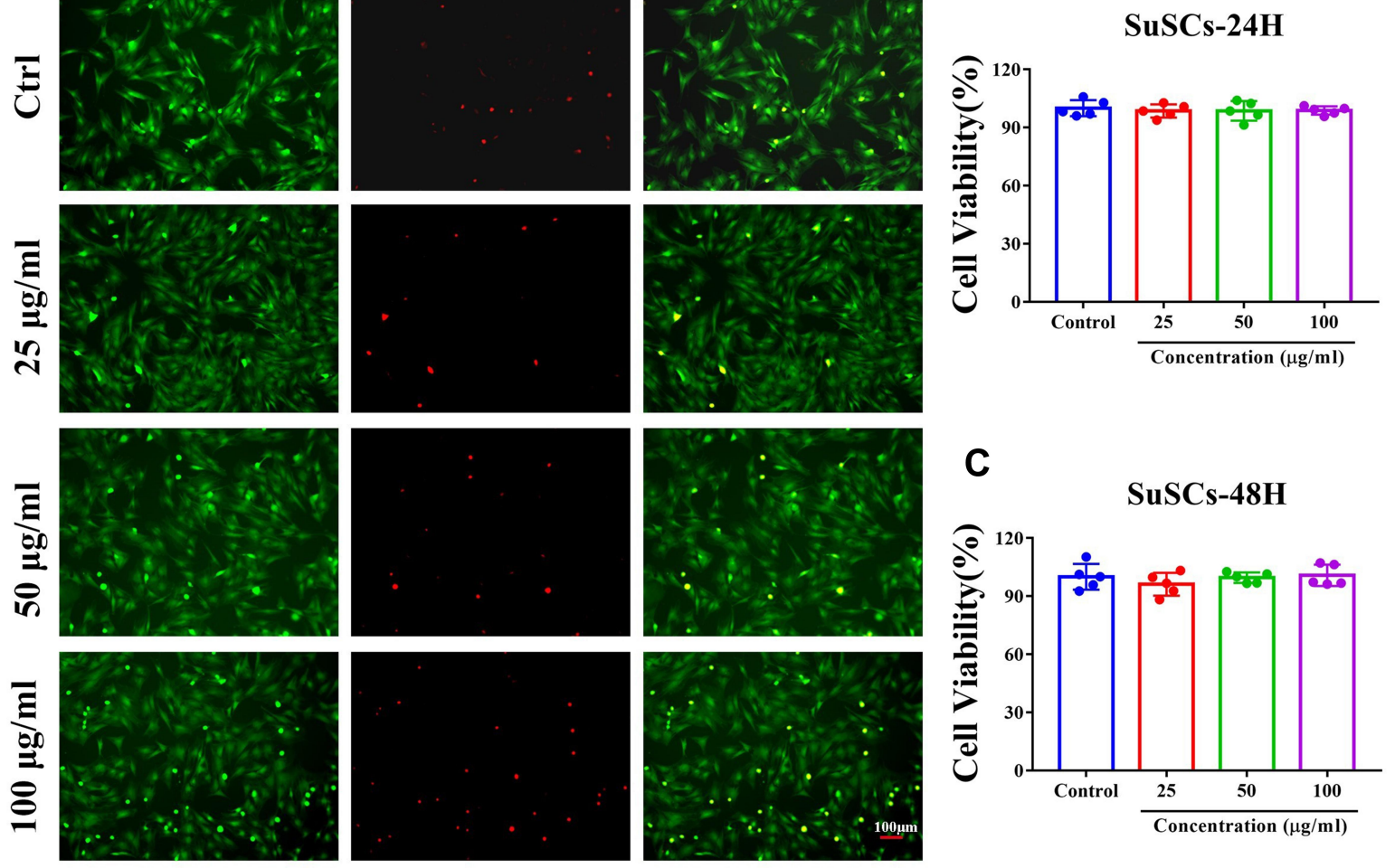

D
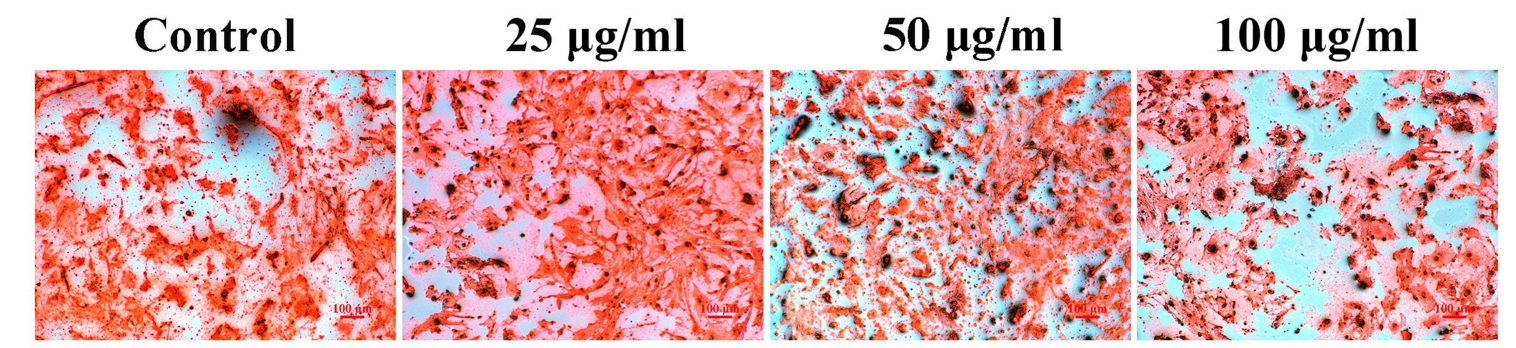

E

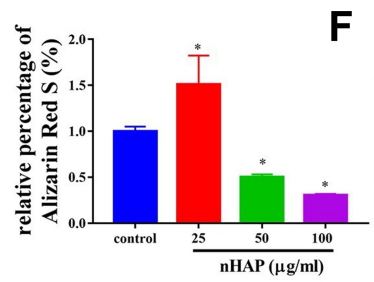

F

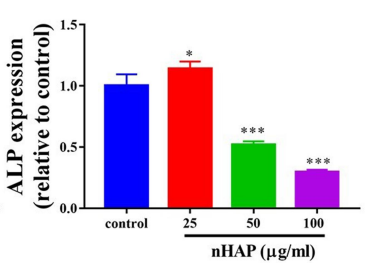

I

G

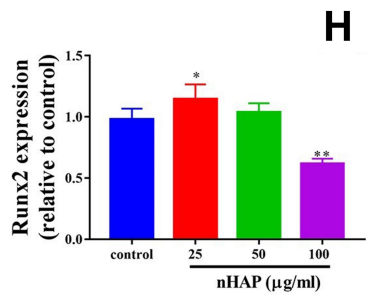

H

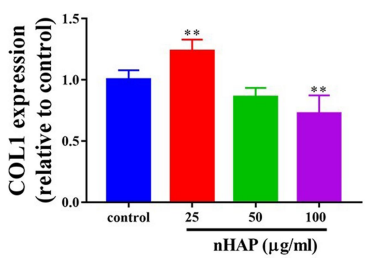

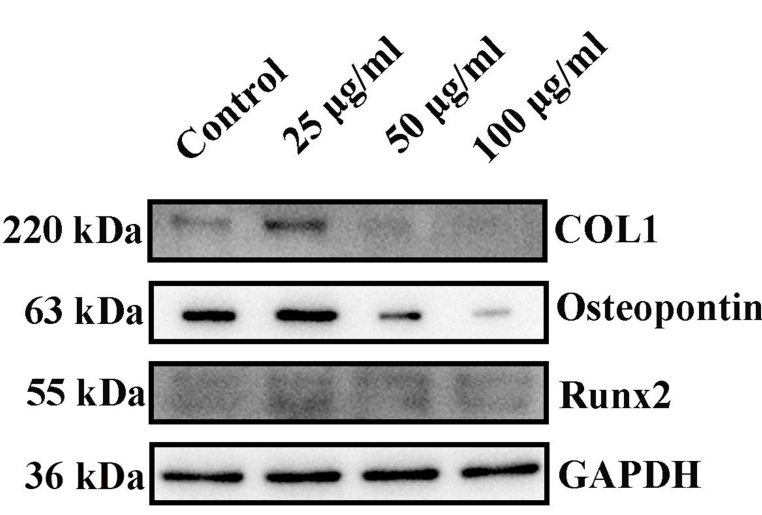

Figure 3 The effects of $\mathrm{nHAP}$ on the viability and osteoblast differentiation of SuSCs. (A) SuSCs were exposed to various concentrations of $\mathrm{nHAP}(0,25,50$ and I00 $\mu \mathrm{g} / \mathrm{mL})$ for $48 \mathrm{~h}$. Live/dead staining was applied to assess the cytotoxicity of nHAP. Scale bar, $100 \mu \mathrm{m}$. (B) CCK-8 analysis evaluates the viability of SuSCs treated with nHAP in different concentrations at $24 \mathrm{~h}$, and (C) $48 \mathrm{~h}$. (D) The extracellular calcium deposition was visualized by Alizarin Red S staining after cells were cultured with nHAP in different concentrations for 14 days; scale bar, $100 \mu \mathrm{m}$. (E) Mineralization was quantified following the colorimetric analysis of Alizarin Red $\mathrm{S}$ elution from calcium nodules. The expression level of osteoblastogenic genes (F) alp, (G) runx2 and $(\mathbf{H})$ coll in the presence of various concentrations of nHAP. *p $<0.05$, **p $<0.0$ I, ***p $<0.00$ I. (I) The expression of osteoblast-associated proteins (coll, runx2, osteopontin) under nHAP treatment was assessed. 
suggested that nHAP has excellent biocompatibility in applications in vivo.

\section{nHAP Strongly Facilitates Osteoblast Differentiation and Matrix Mineralization in SuSCs in Presence of Mechanical Stimuli}

It was reported that concentration of nHAP played an important role in reinforcing osteoblast differentiation. ${ }^{40}$ Therefore, SuSCs were cultured in an osteogenic medium containing increasing concentrations $(0,25,50$ and $100 \mu \mathrm{g} / \mathrm{mL}$ ) of nHAP in the presence of mechanical stimuli to screen for optimal osteoinductive concentration of nHAP. With the treatment of various concentrations of nHAP for 14 days, we conducted Alizarin Red $\mathrm{S}$ staining to assess the mineralization rate (the late stage of osteogenic differentiation) of all groups. As shown in Figure 3D, notably, $25 \mu \mathrm{g} / \mathrm{mL}$ nHAP significantly increased the mineralized matrix depositions after 14 days as compared to the control; whilst with nHAP concentration reached $100 \mu \mathrm{g} / \mathrm{mL}$, lesser mineralized matrix depositions of SuSCs were observed compared with the control group. The mineralization rate, which was quantified following Alizarin Red S elution from the stained mineral nodules, showed that $25 \mu \mathrm{g} / \mathrm{mL}$ nHAP enhanced the mineralization rate $(\mathrm{p}<0.05)$. However, 50 and $100 \mu \mathrm{g} / \mathrm{mL}$ nHAP significantly reduced the mineralization rate as compared to the control group $(\mathrm{p}<0.05$; Figure $3 \mathrm{E})$. Furthermore, the expression levels of osteoblastogenic genes, including alp, col1 and runx2, were examined by RT-qPCR after co-culturing SuSCs in the presence of nHAP for 14 days. As shown in Figures $3 \mathrm{~F}-\mathrm{H}$, the expressions of three osteoblastic genes shared a similar trend with the $25 \mu \mathrm{g} / \mathrm{mL}$ nHAP group showing the highest value $(\mathrm{p}<$ 0.05 as compared with the control group). However, nHAP concentrations approaching $100 \mu \mathrm{g} / \mathrm{mL}$ were observed to induce a significant down-regulation in the three genes as compared with the control group $(0 \mu \mathrm{g} /$ $\mathrm{mL}$ ). Correspondingly, we further identified the role of nHAP in osteoblast differentiation of SuSCs by assessing the expression of osteoblast-associated proteins (Figure 3I). Notably, the expression of coll, runx 2 and osteopontin increased, obviously, at day 14 in the group of $25 \mu \mathrm{g} / \mathrm{mL}$. Meanwhile, the expression of all osteoblast-associated proteins reduced significantly in the $100 \mu \mathrm{g} / \mathrm{mL}$ group. According to the above results, the
nHAP concentration of $25 \mu \mathrm{g} / \mathrm{mL}$ was selected for animal study due to the maximal osteoinductive efficiency on SuSCs, which were consistent with the previous study. ${ }^{41}$

\section{Micro-CT Analysis}

At the end of the study, all animals were alive and healthy. Reconstructed 3D images obtained from the micro-CT are shown in Figure 4A. A significant reduction of bone tissue was observed in the distraction group compared with the SHAM group; the latter represented natural suture width and bone tissue. Notably, a better-organized microarchitecture was consistently found in the expanded sagittal suture treated with ACS+nHAP. After analysis, the suture gap area of the ACS+nHAP group was significantly smaller than that of the distraction group and ACS group ( $\mathrm{p}<$ 0.05 ) and almost restored to the level of the SHAM group $(p>0.05)$. No significant differences in the suture gap areas were detected between the distraction group and the ACS group ( $p>0.05)$ (Figure 4B). Bone formation in the sagittal suture was defined by the changes of bone mineral density (BMD) and bone volume/tissue volume ratio (BV/ TV). In the sagittal suture of the distraction group, the BMD significantly declined, from $3205.25 \pm 53.72$ to $2976.21 \pm 42.56 \mathrm{mg} / \mathrm{cc}(\mathrm{p}<0.05)$. Following treatment with ACS+nHAP, a significant increment in BMD in the ACS +nHAP group occurred, with an increase of 161.18 $\pm 5.23 \mathrm{mg} / \mathrm{cc}(\mathrm{p}<0.05$; Figure 4C), and BMD was almost restored to the level of the SHAM group. Simultaneously, a similar trend was observed in BV/TV. The BV/TV was significantly higher after the ACS+nHAP treatment, with an increment from $6.98 \%$ to $13.16 \%$ ( $<<0.01$; Figure 4D). There were no differences between the ACS + nHAP and SHAM groups, the latter having a BV/TV of $15.14 \%$. All these results indicated the role of nHAP in the facilitation of the ossification in expanded sagittal sutures during expansion by tension force.

To further identify the osteoinductive efficacy of nHAP on SuSCs in vivo, we detected the biomarker (BMP-2) of osteoblasts. Fewer BMP-2 positive cells (marked in brown) could be observed in the distraction group and ACS group; meanwhile, with the nHAP treatment, more obvious brown cells were observed in sagittal sutures. H\&E staining of the sagittal sutures also showed an obvious increase of bone microarchitecture with nHAP treatment when compared with the distraction group (Figure 5). 
A
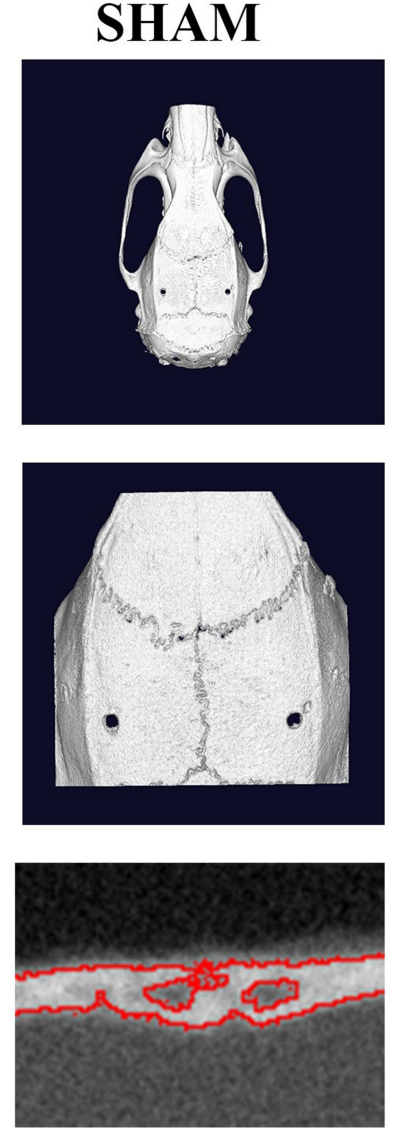

B

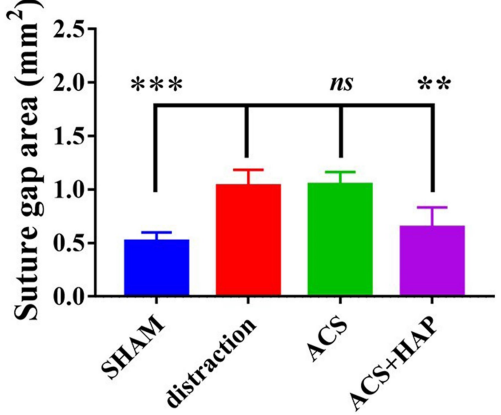

\section{Distraction}
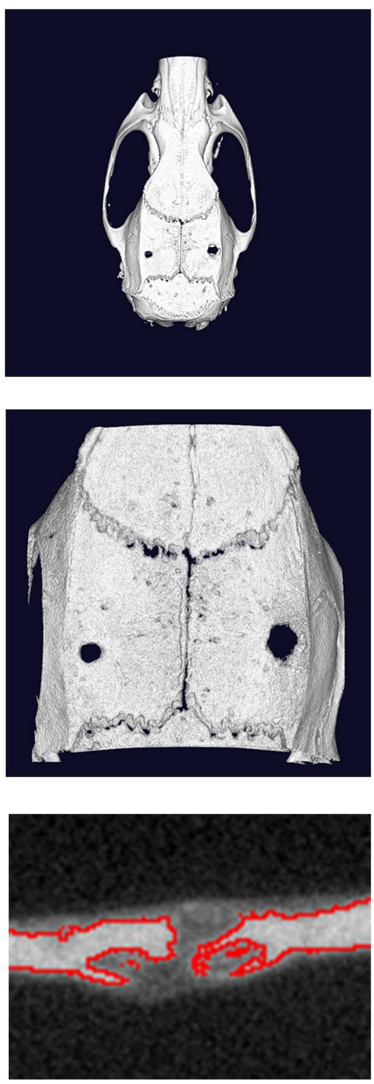

C

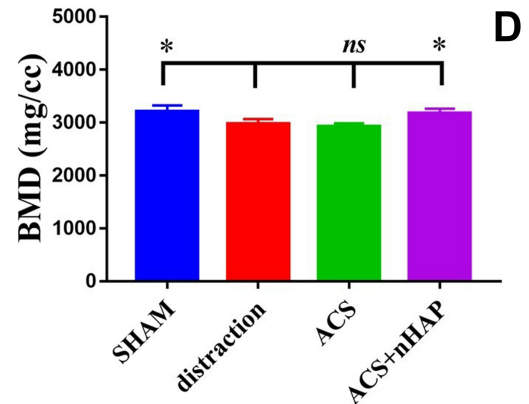

ACS
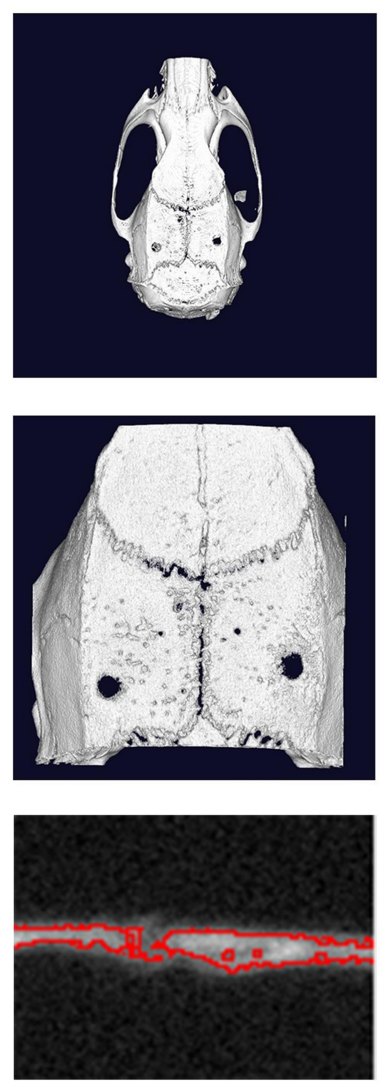

ACS+nHAP
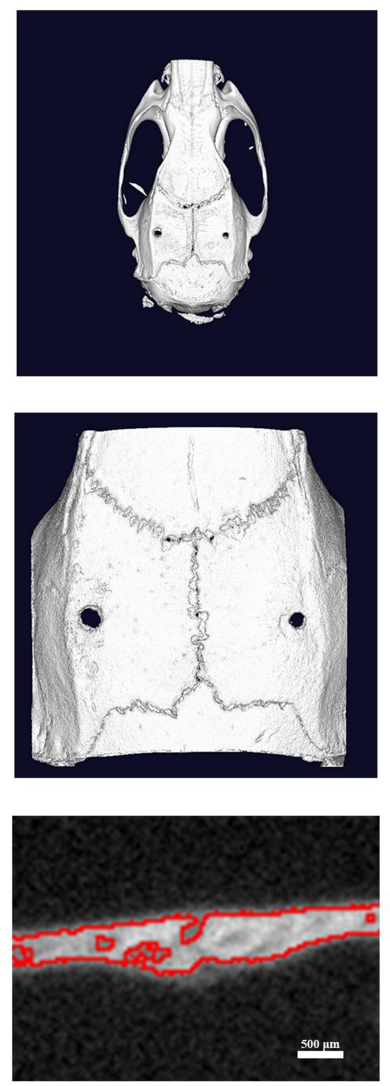

Figure 4 Three-dimensional micro-CT reconstruction of all groups. (A) Micro-CT reconstruction images of calvaria at day 14 after different treatments. A magnified image of the sagittal suture area is shown in the middle panel of each image. Scale bars are $2 \mathrm{~mm}$ in top panel and I mm in middle panel. Coronal planes of sagittal suture areas are viewed in the bottom panel, scale bars $500 \mu \mathrm{m}$. (B) Suture gap area, (C) bone mineral density (BMD) and (D) bone volume fraction (BV/TV) of each sagittal suture. *P < 0.05 , **p $<0.01$, ***p $<0.001$.

\section{Nanoindentation Assessment}

A sufficient mechanical strength of the bone matrix is closely related to relapse rate. Consequently, we also performed a nanoindentation test to reveal the altered mechanical properties of the bone matrix in the sagittal sutures. The nanoindentation test probes the properties at a nanometer length scale, and is a versatile technique for mechanical assaying of a variety of specimens, from soft tissues to bones with impressive spatial resolutions of a few tens to a few hundred nanometer. ${ }^{42}$ In general, nanoindentation provides both elastic modulus and hardness estimates for bone tissues. The mechanical properties of the bone, such as load-displacement curves, elastic modulus, hardness, fracture toughness, strain hardening effect, viscoelasticity, or creep behavior, can be obtained by a needle-like indenter pressed into the surface of the sample. ${ }^{43}$ A total of 34 indents were plotted during the test and load-displacement curves were detected on the osteogenic front of the sutures: 10 in the SHAM group, 8 in the distraction group, 8 in the ACS group, and 8 in the ACS 


\section{SHAM}
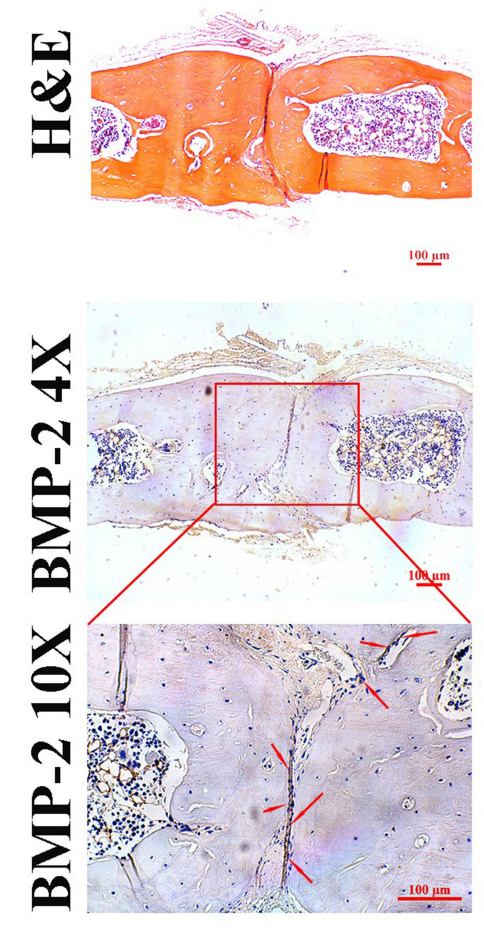

Distraction

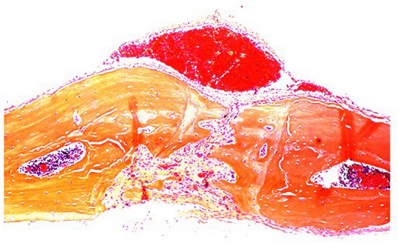

$100 \mu \mathrm{m}$

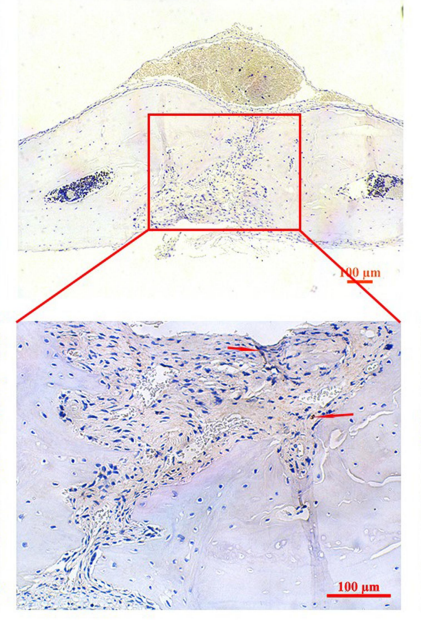

ACS

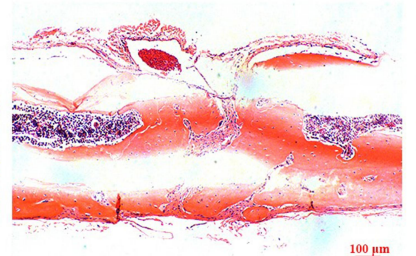

$\underline{100 \mu \mathrm{m}}$

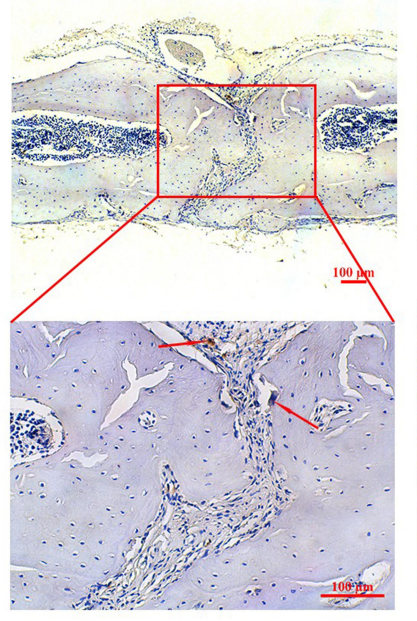

ACS+nHAP
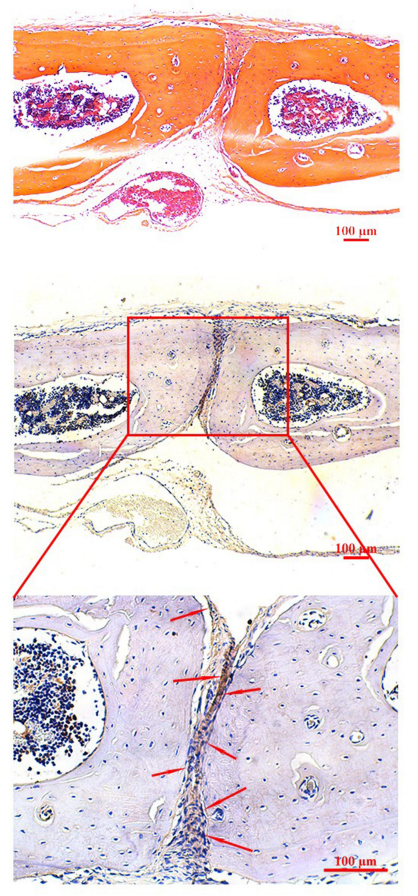

Figure 5 Histochemical staining of sagittal suture tissues from different groups. H\&E staining of sagittal sutures (top panel; scale bar, $100 \mu m$ ). In ACS+nHAP group, the bone microarchitecture developed in a more balanced morphology with homogeneous expansion than distraction group. Immunohistochemistry staining of BMP-2 on sagittal suture tissues (middle and bottom panels) shows that the number of osteoblasts (stained in brown) is more abundant and distributed more homogeneously in the sutures in the ACS + nHAP group than in the other groups. Red arrows indicate osteoblasts. Scale bar, $100 \mu \mathrm{m}$.
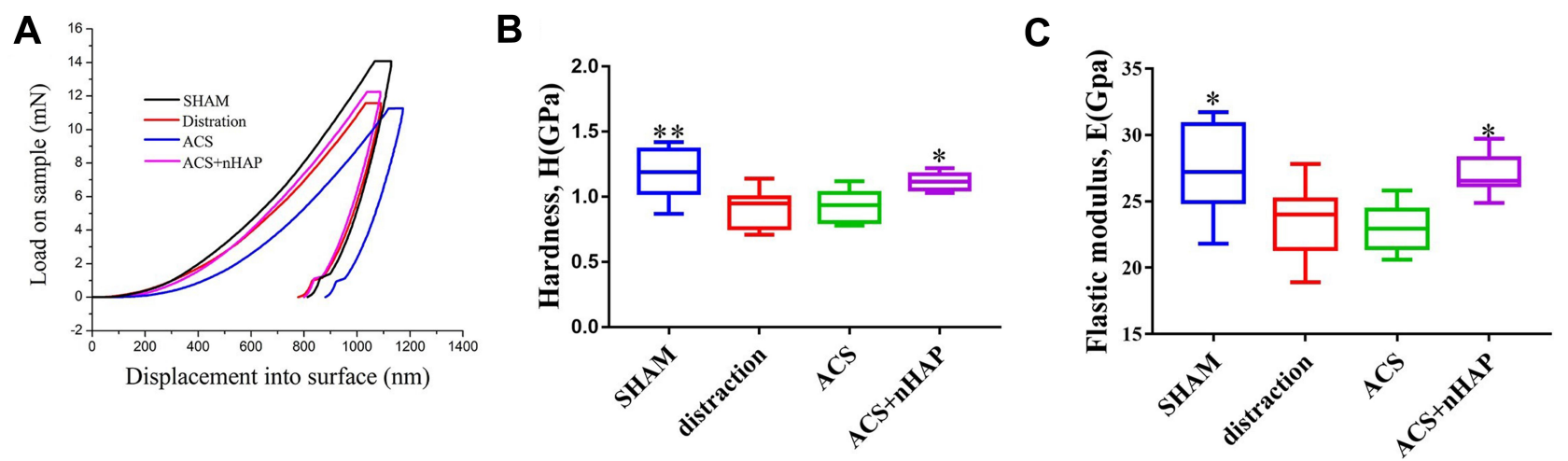

Figure 6 Nanoindentation test assesses the mechanical properties of sagittal suture microarchitecture in various groups. (A) Representative load-displacement curves of each group. (B) Average modulus and (C) Elastic modulus of sagittal suture microarchitecture from various groups. ${ }^{*} \mathrm{p}<0.05$, $* * p<0.01$.

+ nHAP group. The representative curves of loaddisplacement are shown in Figure 6A, and the elastic modulus and hardness were analyzed through the curves. The average elastic modulus of the SHAM group was $27.38 \pm 3.39 \mathrm{GPa}$, while it reduced to $23.61 \pm 2.75 \mathrm{GPa}$ in the distraction group. After the intervention of $25 \mu \mathrm{g} /$ $\mathrm{mL}$ nHAP, the average elastic modulus was increased to $27.08 \pm 1.52 \mathrm{GPa}$. No difference in elastic modulus was observed between the distraction group and the ACS group
(Figure 6B). The hardness presented a similar trend to the elastic modulus with treatment of nHAP (Figure 6C).

\section{Discussion}

The treatment of patients with craniofacial disharmony remains a critical challenge because suture expansion has a high tendency to relapse and requires a retention period of 3 to 9 months. ${ }^{44,45}$ A major reason is the length of time required for the new bone formation in the expanded 
sutural area. Thus, the speed and quality of ossification in the sutural area during expansion can affect the post-treatment relapse rate. ${ }^{46}$ In the current study, we determined that nHAP has a positive effect on bone formation in the expanded rat sagittal sutures. To the best of our knowledge, this research is the first to inspect the effects of nHAP on reducing the relapse rate of expanded sutures via inducing osteoblast differentiation in SuSCs and enhancing local mechanical support.

Actually, an ideal suture expansion approach is composed of the rapid expansion and ossification process. ${ }^{10}$ In terms of the magnitude of stretch force, in the rat model, light forces can slightly induce bone regeneration but cannot maintain the suture expansion permanently. Furthermore, greater amounts of force cannot enhance the bone regeneration but only increase the sutural width and lead to subsequent relapse potential. $^{2,47}$ Therefore, an approximately $50 \mathrm{~g}$ of stretch force was used in this study, which was validated as promoting bone regeneration in previous studies. ${ }^{13,48}$ In addition, 14 days was selected as the experimental period because 14 days in a rat's life is approximately equal to one year in a human's life, which is the average treatment duration of the TSDO technique. ${ }^{49}$

To improve the efficiency and quality of ossification in the expanded suture, investigations of pharmacologic agents or biomaterials with osteoinductive effects have become popular. Generally, most research focuses on evaluating the macroscopical effect of anabolic agents. However, a few studies have assessed the underlying mechanism of anabolic agents on SuSCs, carrying multiple differentiation abilities and intrinsic reparative potential. ${ }^{50}$ Unlike previous research, we investigated the prominent osteoinductive effect and the excellent bioactivity of nHAP on SuSCs and defined the optimal induction concentration of nHAP necessary for osteoblast differentiation in SuSCs. Based on the results of cellular research, a direct analysis of nHAP for subsequent pre-clinical application was performed. With respect to the underlying mechanisms, the osteoinductive effects and bioinorganic material features of nHAP may account for the results. On the one hand, as the initiator of bone formation, nHAP could induce SuSC differentiation and enhance osteoblast activity by supplying enough calcium and phosphate surrounding SuSCs to result in new bone regeneration. ${ }^{51,52}$ Due to the nano-scaled feature of underlying crystals, compared to conventional HAP with a flat surface, nHAP produces a higher degree of stress concentration at the cellcrystal interface, and a higher extension of cells on the nHAP surface enhances $\mathrm{Ca}^{2+}$ exchange, which favors osteoblast differentiation of MSCs. ${ }^{53,54}$ Moreover, the nHAP can mediate the adhesion of specific anchoragedependent cells by adsorbing extracellular matrix protein, including fibronectin, and growth factors, like osteonectin. ${ }^{39}$ Interestingly, several studies reported that autophagy in osteoblasts is involved both in mineralization and bone homeostasis and defined nHAP as a novel class of autophagy inducer. Moreover, it is not the material species but in fact the size or shape that stimulates autophagy. Wang et al found that lower concentrations of nano-HAP induced moderate cell autophagy and promoted osteoblast differentiation via the PI3K/Akt/mTOR pathway. ${ }^{55}$ Collectively, these mechanisms could differentially but synergistically account for the reduced relapse ratio administered by nHAP.

Additionally, previous studies performed the BMD (bone mineral density) or BV/TV measurement soley by micro-CT. Remarkably, mechanical strength is another inescapable parameter of bone quality. ${ }^{56}$ Thus, our innovation in this work is application of the nanoindentation test. This test is a new technology for investigating the micromechanical properties of bone tissue. Unlike conventional techniques, nanoindentation provides both elastic modulus and hardness estimates for various bone tissue structures at a microscopic level. ${ }^{42}$ Hardness, a particularly interesting mechanical property of bone, can be extracted from the experimental load-depth curves that are recorded during the indentation test. ${ }^{57}$ Nanoindentation elastic modulus of bone arises mainly from the collagen fiber matrix, and a higher elastic modulus of bone can be an effective indicator of more. ${ }^{58}$ Our nanoindentation test showed improved hardness following the intervention of nHAP compared with the control group. This result indicated that nHAP contributed to the new bone regeneration and strength reinforcement in sutures during the expansion process, which is consistent with the results of micro-CT.

\section{Conclusion}

In summary, we evaluated the osteoinductive effect of nHAP in vitro and in vivo. The results suggested that the application of nHAP (approximately $55 \mathrm{~nm}$ in diameter) can induce osteoblast differentiation in suture-derived stem cells (SuSCs) and stimulate bone regeneration in an expanded sagittal suture during expansion, resulting in the increment of local mechanical strength and reduction of the relapse ratio.

\section{Abbreviations}

TSDO, trans-sutural distraction osteogenesis; SuSCs, calvarial suture-derived stem cells; BMD, bone mineral 
density; EDTA, ethylene diaminetetra acetic acid; H\&E, hematoxylin and eosin; MSCs, mesenchymal stem cells; ALP, Alkaline phosphatase; Runx2, RUNX family transcription factor 2; COL1, Collagen type I ; nHAP, hydroxyapatite nanoparticle; SHAM, sham-surgery; ACS, absorbable collagen sponge; BV/TV, bone volume fraction; BMP-2, bone morphogenetic protein-2; CTAB, Hexadecyl (cetyl) trimethyl ammonium bromide; DLS, dynamic light scattering; XRD, X-ray diffraction; FACS, fluorescent-activated cell sorting; CLSM, confocal laser scanning microscope; PI, propidium iodide.

\section{Acknowledgments}

This project was supported by the National Natural Science Funder of China (No. 81571925). We are thankful for the technical support provided by the Animal Experiment Center of Peking University Health Science Center. We thank Professor YI Weining from the Department of Public Health, Peking University Health Science Center, who provided guidance on the statistical methods used, and Hung Kanlin, who helped edit and polish our manuscript carefully.

\section{Author Contributions}

All authors made substantial contributions to conception and design, acquisition of data, or analysis and interpretation of data; took part in drafting the article or revising it critically for important intellectual content; agreed to submit to the current journal; gave final approval of the version to be published; and agree to be accountable for all aspects of the work.

\section{Disclosure}

The authors report no conflicts of interest for this work and declare no competing financial interests.

\section{References}

1. Tong H, Wang X, Song T, et al. Trans-sutural distraction osteogenesis for midfacial hypoplasia in growing patients with cleft lip and palate. Plast Reconstr Surg. 2015;136(1):144-155.

2. Takeshita N, Hasegawa M, Sasaki $\mathrm{K}$, et al. In vivo expression and regulation of genes associated with vascularization during early response of sutures to tensile force. J Bone Miner Metab. 2017;35 (1):40-51.

3. Hou B, Fukai N, Olsen BR. Mechanical force-induced midpalatal suture remodeling in mice. Bone. 2007;40(6):1483-1493.

4. Wu BH, et al. Stretch force guides finger-like pattern of bone formation in suture. PLoS One. 2017;12(5):e0177159.

5. Liu Y, Tang Y, Xiao L, Liu SS, Yu H. Suture cartilage formation pattern varies with different expansive forces. Am $J$ Orthod Dentofacial Orthop. 2014;146(4):442-450.
6. Matsugaki A, Fujiwara N, Nakano T. Continuous cyclic stretch induces osteoblast alignment and formation of anisotropic collagen fiber matrix. Acta Biomater. 2013;9(7):7227-7235.

7. Zhao S, et al. Effects of strontium ranelate on bone formation in the mid-palatal suture after rapid maxillary expansion. Drug Des Devel Ther. 2015;9:2725-2734.

8. Ozturk F, Wang X, Li N, Chen Y, Su Y, Zhang J. Effects of bisphosphonates on sutural bone formation and relapse: a histologic and immunohistochemical study. Am J Orthod Dentofacial Orthop. 2011;140(1):e31-41.

9. Koons GL, Diba M, Mikos AG. Materials design for bone-tissue engineering. Nat Rev Mater. 2020;5(8):584-603.

10. Liu SS, Xu H, Sun J, et al. Recombinant human bone morphogenetic protein-2 stimulates bone formation during interfrontal suture expansion in rabbits. Am J Orthod Dentofacial Orthop. 2013;144(2):210-217.

11. Uysal T, Amasyali M, Olmez H, et al. Effect of vitamin C on bone formation in the expanded inter-premaxillary suture. Early bone changes. J Orofac Orthop. 2011;72(4):290-300.

12. Bulut M, Korkmaz YN, Erimsah S. Effects of isotretinoin on new bone formation after maxillary sutural expansion. J Orofac Orthop. 2020;81(6):440-446.

13. Yi J, Mei L, Li X, Zheng W, Li Y, Zhao Z. Effects of continuous and intermittent parathyroid hormone administration on midpalatal suture expansion in rats. Arch Oral Biol. 2019;99:161-168.

14. Ekizer A, Yalvac ME, Uysal T, Sonmez MF, Sahin F. Bone marrow mesenchymal stem cells enhance bone formation in orthodontically expanded maxillae in rats. Angle Orthod. 2015;85(3):394-399.

15. Quan H, He Y, Sun J, et al. Chemical self-assembly of multifunctional hydroxyapatite with a coral-like nanostructure for osteoporotic bone reconstruction. ACS Appl Mater Interfaces. 2018;10(30):25547-25560.

16. Zhao R, Xie P, Zhang K, et al. Selective effect of hydroxyapatite nanoparticles on osteoporotic and healthy bone formation correlates with intracellular calcium homeostasis regulation. Acta Biomater. 2017;59:338-350.

17. El-Rashidy AA, Roether JA, Harhaus L, et al. Regenerating bone with bioactive glass scaffolds: a review of in vivo studies in bone defect models. Acta Biomater. 2017;62:1-28.

18. Zhao R, Chen S, Yuan B, et al. Healing of osteoporotic bone defects by micro-/nano-structured calcium phosphate bioceramics. Nanoscale. 2019;11(6):2721-2732.

19. Maeno S, Niki Y, Matsumoto H, et al. The effect of calcium ion concentration on osteoblast viability, proliferation and differentiation in monolayer and 3D culture. Biomaterials. 2005;26(23):4847-4855.

20. Ha SW, Jang HL, Nam KT. Nano-hydroxyapatite modulates osteoblast lineage commitment by stimulation of DNA methylation and regulation of gene expression. Biomaterials. 2015;65:32-42.

21. Shi Z, Huang X, Cai Y. Size effect of hydroxyapatite nanoparticles on proliferation and apoptosis of osteoblast-like cells. Acta Biomater. 2009;5(1):338-345.

22. Dos Anjos S, Mavropoulos E, Alves GG, et al. Impact of crystallinity and crystal size of nanostructured carbonated hydroxyapatite on pre-osteoblast in vitro biocompatibility. $J$ Biomed Mater Res A. 2019;107(9):1965-1976.

23. Zhao Y, Zhang Y, Ning F. Synthesis and cellular biocompatibility of two kinds of HAP with different nanocrystal morphology. J Biomed Mater Res B Appl Biomater. 2007;83(1):121-126.

24. Maruyama T, Jeong J, Sheu TJ. Stem cells of the suture mesenchyme in craniofacial bone development, repair and regeneration. Nat Commun. 2016;7:10526.

25. Doro DH, Grigoriadis AE, Liu KJ. Calvarial suture-derived stem cells and their contribution to cranial bone repair. Front Physiol. 2017;8:956.

26. James AW, Levi B, Xu Y. Retinoic acid enhances osteogenesis in cranial suture-derived mesenchymal cells: potential mechanisms of retinoid-induced craniosynostosis. Plast Reconstr Surg. 2010;125 (5):1352-1361. 
27. Takahashi Y, Mayahara K, Fushiki R. Effect of mechanical strain-induced PGE2 production on bone nodule formation by rat calvarial progenitor cells. J Oral Sci. 2019;61(1):25-29.

28. Song D, Zhang F, Reid RR, et al. BMP9 induces osteogenesis and adipogenesis in the immortalized human cranial suture progenitors from the patent sutures of craniosynostosis patients. J Cell Mol Med. 2017;21(11):2782-2795.

29. Ikegame M, Ejiri S, Okamura H. Expression of non-collagenous bone matrix proteins in osteoblasts stimulated by mechanical stretching in the cranial suture of neonatal mice. J Histochem Cytochem. 2019;67 (2):107-116

30. Liu Y, Wang G, Cai Y, et al. In vitro effects of nanophase hydroxyapatite particles on proliferation and osteogenic differentiation of bone marrow-derived mesenchymal stem cells. J Biomed Mater Res A. 2009;90(4):1083-1091.

31. Dome K, Podgorbunskikh E, Bychkov A, Lomovsky O. Changes in the crystallinity degree of starch having different types of crystal structure after mechanical pretreatment. Polymers (Basel). 2020;12(3).

32. James AW, Xu Y, Wang R, Longaker MT. Proliferation, osteogenic differentiation, and fgf-2 modulation of posterofrontal/sagittal suture-derived mesenchymal cells in vitro. Plast Reconstr Surg. 2008;122(1):53-63.

33. Chen K, Geng H, Liang W, et al. Modulated podosome patterning in osteoclasts by fullerenol nanoparticles disturbs the bone resorption for osteoporosis treatment. Nanoscale. 2020;12(17):9359-9365.

34. Dominici M, Le Blanc K, Mueller I, et al. Minimal criteria for defining multipotent mesenchymal stromal cells. The international society for cellular therapy position statement. Cytotherapy. 2006;8 (4):315-317.

35. Sun L, Qu L, Zhu R, et al. Effects of mechanical stretch on cell proliferation and matrix formation of mesenchymal stem cell and anterior cruciate ligament fibroblast. Stem Cells Int. 2016;20 16:9842075.

36. Huang Z, Cheng C, Cao B, et al. Icariin protects against glucocorticoid-induced osteonecrosis of the femoral head in rats. Cell Physiol Biochem. 2018;47(2):694-706.

37. Lawson MA, Xia Z, Barnett BL, et al. Differences between bisphosphonates in binding affinities for hydroxyapatite. $J$ Biomed Mater Res B Appl Biomater. 2010;92(1):149-155.

38. Kim M, Steinberg DR, Burdick JA. Extracellular vesicles mediate improved functional outcomes in engineered cartilage produced from MSC/chondrocyte cocultures. Proc Natl Acad Sci U S A. 2019;116 (5):1569-1578.

39. Guo X, Gough JE, Xiao P. Fabrication of nanostructured hydroxyapatite and analysis of human osteoblastic cellular response. J Biomed Mater Res A. 2007;82(4):1022-1032.

40. Wang L, Zhou G, Liu H, et al. Nano-hydroxyapatite particles induce apoptosis on MC3T3-E1 cells and tissue cells in SD rats. Nanoscale. 2012;4(9):2894-2899.

41. Balcerzak M, Hamade E, Zhang L. The roles of annexins and alkaline phosphatase in mineralization process. Acta Biochim Pol. 2003;50 (4):1019-1038.

42. Nautiyal P, Alam F, Balani K. The role of nanomechanics in healthcare. Adv Healthc Mater. 2018;7(3).
43. Luo C, Liao J, Zhu Z. Analysis of mechanical properties and mechanical anisotropy in canine bone tissues of various ages. Biomed Res Int. 2019;2019:3503152.

44. Petrick S, Hothan T, Hietschold V. Bone density of the midpalatal suture 7 months after surgically assisted rapid palatal expansion in adults. Am J Orthod Dentofacial Orthop. 2011;139(4 Suppl):S109_ 16.

45. Liou EJ, Tsai WC. A new protocol for maxillary protraction in cleft patients: repetitive weekly protocol of alternate rapid maxillary expansions and constrictions. Cleft Palate Craniofac J. 2005;42 (2):121-127.

46. Saito S, Shimizu N. Stimulatory effects of low-power laser irradiation on bone regeneration in midpalatal suture during expansion in the rat. Am J Orthod Dentofacial Orthop. 1997;111(5):525-532.

47. Fushiki R. High-magnitude mechanical strain inhibits the differentiation of bone-forming rat calvarial progenitor cells. Connect Tissue Res. 2015;56(4):336-341.

48. Liu SS, Kyung HM, Buschang PH. Continuous forces are more effective than intermittent forces in expanding sutures. Eur J Orthod. 2010;32(4):371-380.

49. Quinn R. Comparing rat's to human's age: how old is my rat in people years? Nutrition. 2005;21(6):775-777.

50. Wilk K. Postnatal calvarial skeletal stem cells expressing PRX1 reside exclusively in the calvarial sutures and are required for bone regeneration. Stem Cell Rep. 2017;8(4):933-946.

51. Bodhak S, Bose S, Bandyopadhyay A. Role of surface charge and wettability on early stage mineralization and bone cell-materials interactions of polarized hydroxyapatite. Acta Biomater. 2009;5 (6):2178-2188.

52. MacMillan AK. Similar healthy osteoclast and osteoblast activity on nanocrystalline hydroxyapatite and nanoparticles of tri-calcium phosphate compared to natural bone. Int J Nanomedicine. 2014; 9:5627-5637.

53. Balasundaram G, Sato M, Webster TJ. Using hydroxyapatite nanoparticles and decreased crystallinity to promote osteoblast adhesion similar to functionalizing with RGD. Biomaterials. 2006;27(14): 2798-2805.

54. Hesaraki S. Comparative study of mesenchymal stem cells osteogenic differentiation on low-temperature biomineralized nanocrystalline carbonated hydroxyapatite and sintered hydroxyapatite. J Biomed Mater Res B Appl Biomater. 2014;102(1):108-118.

55. Wang R. Nano-hydroxyapatite modulates osteoblast differentiation through autophagy induction via mTOR signaling pathway. J Biomed Nanotechnol. 2019;15(2):405-415.

56. Rachner TD, Khosla S, Hofbauer LC. Osteoporosis: now and the future. Lancet. 2011;377(9773):1276-1287.

57. Molino G. Osteoporosis-related variations of trabecular bone properties of proximal human humeral heads at different scale lengths. J Mech Behav Biomed Mater. 2019;100:103373.

58. Isaksson H, Malkiewicz M, Nowak R, Helminen HJ, Jurvelin JS. Rabbit cortical bone tissue increases its elastic stiffness but becomes less viscoelastic with age. Bone. 2010;47(6):1030-1038.
Drug Design, Development and Therapy

\section{Publish your work in this journal}

Drug Design, Development and Therapy is an international, peerreviewed open-access journal that spans the spectrum of drug design and development through to clinical applications. Clinical outcomes, patient safety, and programs for the development and effective, safe, and sustained use of medicines are a feature of the journal, which has also been accepted for indexing on PubMed Central. The manuscript management system is completely online and includes a very quick and fair peer-review system, which is all easy to use. Visit http://www. dovepress.com/testimonials.php to read real quotes from published authors. 